\title{
Percutaneous device closure of a pseudoaneurysm of the left ventricular wall
}

\author{
P Clift, S Thorne, J de Giovanni
}

Heart 2004;90:e62 (http://www.heartjnl.com/cgi/content/full/90/10/e62). doi: 10.1136/hrt.2004.042135

The percutaneous device closure of a left ventricular pseudoaneurysm is described in a 60 year old man with a history of myocardial infarction complicated by ventricular tachycardia and left ventricular aneurysm treated by coronary artery bypass grafting and aneursymectomy with ventricular tachycardia ablation. He subsequently developed a vast pseudoaneurysm of the left ventricle with New York Heart Association functional class II heart failure symptoms. The selection of the approach and type of device used to close the neck of the pseudoaneurysm are discussed.

W e present the case of a 60 year old man who sustained a myocardial infarction, which was complicated by a left ventricular (LV) aneurysm and ventricular tachycardia, and underwent coronary bypass grafting and LV aneurysmectomy in 1999 together with open mapping and ablation. One year later he developed methicillin resistant Staphylococcus aureus (MRSA) septicaemia and a left sided empyema, which required surgical decortication through a left thoracotomy in late 2000. He presented after an abnormal cardiac silhouette was found on routine chest radiography. Symptomatically he had New York Heart Association (NYHA) functional class II heart failure with no anginal symptoms. Examination showed atrial fibrillation, a dynamic LV apical impulse, and an apical pansystolic murmur. ECG showed atrial fibrillation Q waves in leads VIV4 and a normal QRS axis. Transthoracic echocardiography (TTE) and magnetic resonance angiography (MRA) showed a large pseudoaneurysm with a high velocity bidirectional jet between the posterior wall of the LV close to the apex and a vast sump. It was felt that surgery carried a high risk but a percutaneous approach was feasible. We believe this is the first report of such a procedure.

\section{CASE REPORT}

The procedure was performed under local anaesthetic and fluoroscopic guidance with TTE in the catheter laboratory. A right brachial artery cut down approach was used, as the delivery catheter length precluded a femoral approach. TTE showed the position of the orifice to the pseudoaneurysm in relation to the surgical patch. A 6 French pigtail catheter was advanced into the LV cavity; angiography confirmed a communication arising from the posteroapical portion of the LV cavity into a large false aneurysm occupying a large space in the left pleural cavity (fig 1). A floppy Terumo guidewire (Terumo Corp, Tokyo, Japan) was passed into the aneurysm cavity through the communicating orifice and pressure tracings were made ( $\mathrm{LV} 85 / 0-5 \mathrm{~mm} \mathrm{Hg}$, aneurysm cavity 63/14 mm Hg). Hand contrast angiography confirmed position. A 0.035 inch J tipped exchange wire was passed into the aneurysm cavity over which was passed a $24 \mathrm{~mm}$ Amplatzer sizing balloon (AGA Medical Corp, Golden
Valley, Minnesota, USA), which sized the orifice at $12 \mathrm{~mm}$ and occluded the defect on colour flow mapping. A 9 French Amplatzer sheath was passed through the defect and a $12 \mathrm{~mm}$ Amplatzer septal occluder was deployed across the defect in the usual manner under echocardiographic and fluoroscopic guidance. No residual flow was seen on colour flow mapping. LV angiography confirmed a stable device position (fig 2). A trivial amount of contrast was seen in the aneurysmal space. The patient returned to the ward without complication.

TTE and chest radiography the next day confirmed a well seated device and clot within the aneurysm cavity but with no residual shunt. At follow up he remained well in NYHA class I, and TTE and transoesophageal echocardiography showed a tethered posterior mitral valve leaflet and a normally functioning anterior mitral valve leaflet with a very eccentric jet of mitral incompetence of moderate severity. There was trivial aortic incompetence. The pseudoaneurysm was seen with no flow into the cavity.

\section{DISCUSSION}

Pseudoaneurysm of the LV cavity has been reported after myocardial infarction and myocardial surgery. ${ }^{1-3}$ It is associated with mitral regurgitation and a high mortality. ${ }^{4}$ Conventional surgery carries significant mortality, especially when combined with mitral valve replacement; and medical treatment carries an even higher mortality. ${ }^{2}$ Percutaneous device closure of holes in the heart-for example, atrial and ventricular septal defects-is a recent development that is very widely used and is both safe and effective. A percutaneous approach for treating LV pseudoaneurysm has not been previously reported. Several devices are available including the STARFlex (NMT Medical Inc, Boston, Massachusetts, USA), Helex (WL Gore \& Associates, Flagstaff, Arizona, USA), and Amplatzer (AGA Medical Corp). We considered a transcatheter approach to close the orifice to the aneurysm, as its size and position as seen on the MRA appeared suitable for such an approach. Having considered the common devices available, we elected to implant an Amplatzer septal occluder on the basis of wide experience, robust design, retrievability, and ability to reposition the device. Moreover, the sheath diameter required was relatively small.

There are several Amplatzer devices all based on the same principle and made of the same materials but designed for closure of various defects including atrial septal defect, patent foramen ovale, persistent ductus arteriosus, and muscular, post-myocardial infarction and perimembranous ventricular septal defects. As the orifice to the pseudoaneurysm was at the site of the surgical patch, the thickness of the material was less than that of the neighbouring myocardium; we

Abbreviations: LV, left ventricular; MRA, magnetic resonance angiography; NYHA, New York Heart Association; TE, transthoracic echocardiography 


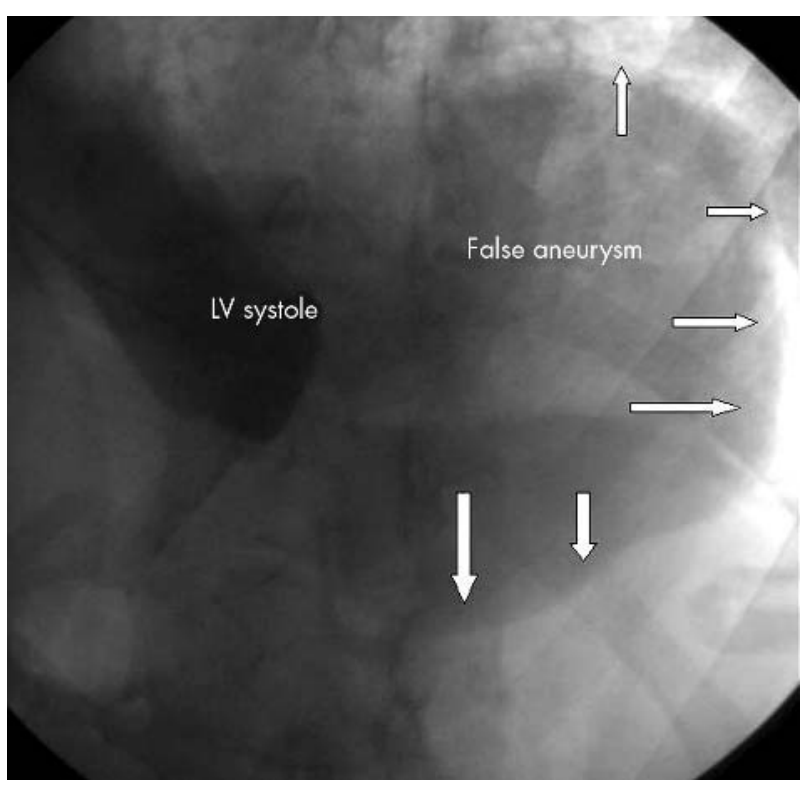

Figure 1 Left ventricular (LV) angiogram in systole, showing the large false aneurysm (arrows).

therefore decided to use an Amplatzer septal occluder, as the length of the central portion between the discs is only $4 \mathrm{~mm}$. This was considered essential for stability and to achieve complete closure.

The MRA clearly showed bidirectional flow between the LV and the pseudoaneurysm. One concern with the interventional approach was that, after the device was deployed, flow from the LV to the aneurysm would persist through the porous device. However, the aneurysm could not generate enough pressure for flow into the LV, thus further enlarging the false aneurysm. This was monitored on TTE during the procedure before the device was released.

The brachial artery approach was chosen, as the length of the sizing balloon and the delivery system are not sufficient for a femoral artery approach. An alternative would have been a femoral vein approach with trans-septal puncture, but the site of the aneurysm orifice would not have favoured this technique.

This approach avoided repeat sternotomy and cardiopulmonary bypass and was performed under local anaesthetic thus reducing the significant morbidity associated with surgery. The patient had a short hospital stay and was well

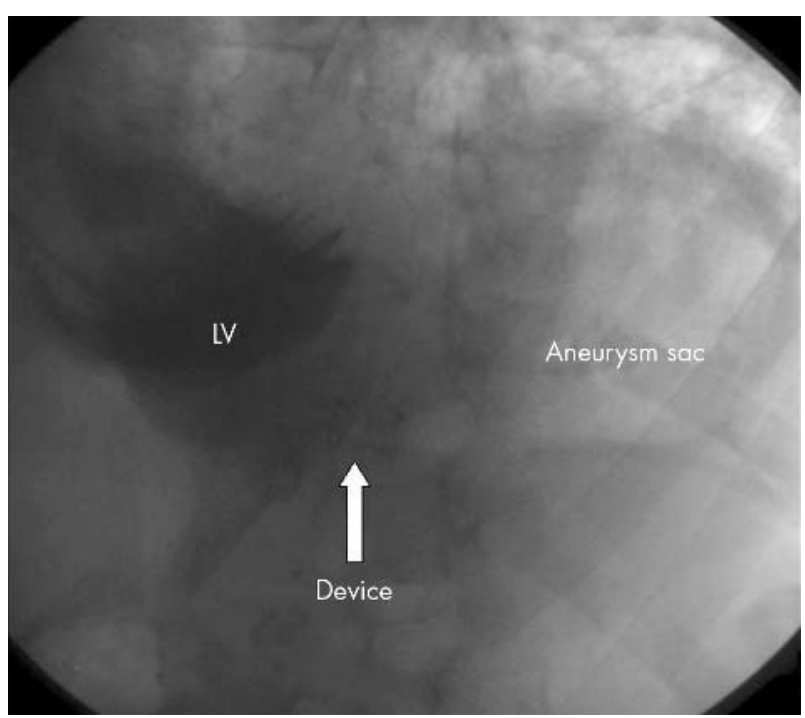

Figure $2 \mathrm{LV}$ angiogram in systole after device closure of the neck of the false aneurysm. The arrow indicates the position of the Amplatzer device.

one year after the procedure. We recommend this approach in selected cases.

\section{Authors' affiliations}

P Clift, S Thorne, Grown Up Congenital Heart Disease Unit, Queen Elizabeth Hospital, University Hospital Birmingham, Birmingham, UK J de Giovanni, Department of Cardiology, Birmingham Children's Hospital, Birmingham, UK

Correspondence to: Dr Jo de Giovanni, Birmingham Children's Hospital, Steelhouse Lane, Birmingham B4 6NH, UK; degiovanni@ blueyonder.co.uk

Accepted 24 May 2004

\section{REFERENCES}

1 Muller I, Andrassy P, Firschke C. Left ventricular pseudoaneurysm: a mechanical complication of acute myocardial infarction. Heart 2002;7:569.

2 Frances C, Romero A, Grady D. Left ventricular pseudoaneurysm. J Am Coll Cardiol 1998;32:557-61.

3 Dubel HP, Rutsch W, Bohm J, et al. Huge false aneurysm of left ventricular posterior wall following resection of an aneurysm of the left ventricular posterior wall. Catheter Cardiovasc Interv 1999;46:509-10.

4 De Paulis R, Zeitani J, Bognolo G, et al. Left ventricular pseudoaneurysm and mitral valve regurgitation: conservative surgical therapy. J Cardiovasc Surg 1999;40:679-81. 